\title{
An improved multivariate chart using partial least squares with continuous ranked probability score
}

\author{
Fouzi Harrou, Member, IEEE, Ying Sun, Muddu Madakyaru and Benamar Bouyedou
}

\begin{abstract}
Reliable fault detection systems in industrial processes provide pertinent information for improving the safety and process reliability and reducing manpower costs. Here, we present a flexible and efficient fault detection approach based on the continuous ranked probability score (CRPS) metric to detect faults in multivariate data. This approach merges partial least squares (PLS) models and the CRPS metric to separate normal from abnormal features by simultaneously taking advantage of the feature representation ability of a PLS and the fault detection capacity of a CRPS-based scheme. The proposed approach uses PLS to generate residuals, and then apply the CRPS-based chart to reveal any abnormality. Specifically, two monitoring schemes based on CRPS measure have been introduced in this paper. The first approach uses the Shewhart scheme to evaluate the CRPS of the response variables residuals from the PLS model. The second approach merges the CRPS into the exponentially weighted moving average monitoring chart. We assess the effectiveness of these approaches by using real and simulated distillation column data. We also compare the detection quality of PLSbased CRPS charts to that of PLS-based $T^{2}, Q$, multivariate cumulative sum, and multivariate exponentially weighted moving average methods. Results show that the capacity of the proposed scheme can reliably detect faults in multivariate processes.
\end{abstract}

Index Terms-Anomaly detection, Multivariate control chart, CRPS, Similarity metrics.

\section{INTRODUCTION}

$\mathbf{F}$ AULT detection in industrial processes is a key enabler for enhancing safety and process reliability, improving profitability and reducing manpower costs [1]-[5]. The main goals of process monitoring are to efficiently detect unacceptable process degradation and to identify the source of abnormality. Multivariate statistical process monitoring methods have been intensively used in process industries to accomplish these objectives [2], [6]. Monitoring methods can provide a warning of unusual changes in the inspected process, and help to identify the onset of potential process or sensor faults. This paper focuses on fault detection tasks, particularly in the monitoring of auto-correlated processes.

Advances made in the areas of online instrumentation and data acquisition have made it possible to collect large amounts of data in the chemical process industry. Given that the data have a certain level of redundancy, it becomes

F. Harrou and Y. Sun are with King Abdullah University of Science and Technology (KAUST) Computer, Electrical and Mathematical Sciences and Engineering (CEMSE) Division, Thuwal, 23955-6900, Saudi Arabia e-mail: fouzi.harrou@kaust.edu.sa

M. Madakyaru is with Manipal Institute of Technology, Department of Chemical Engineering, Manipal University, Manipal, India

B. Bouyedou is with Abou Bekr Belkaid University, Department of Engineering and Telecommunications, Tlemcen, Algeria possible to detect any abnormality in the process. Over the past four decades, several fault detection techniques have been developed [4], [7]-[9]. They are classified into two main categories: those using a data-based approach and those using a model-based approach [10], [11]. The basic philosophy behind model-based approaches is comparing the measured data from a monitored process with analytically computed outputs, and gives a signal when significant differences are identified [4]. Model-based statistical approaches for process monitoring have produced good results in practice [2], [12][16]. However, these approaches depend on the accuracy of the model used. In the absence of analytical models, data-driven approaches can be good alternatives for process monitoring [14], [17]. Some data-based approaches use empirical models constructed by analyzing available data [2], [12], [18]-[20]; others rely on machine learning techniques, such as support vector machine [5], fuzzy inference-based fault detection schemes [21], and random forests classifiers [22]. Data-based methods rely on the availability of quality input data, and their implementation is no easy task, especially for real-time applications.

The purpose of multivariate data-based techniques is to analyze high dimensional data to extract feature information via unmeasurable variables (latent variables) [23]. Indeed, due to dependency and collinearity factors, variations are, for the most part, by only a small number of latent variables termed principal component. For example, the monitoring of chemical process industries involves a large set of measurements that lead to a large amount of redundant information resulting in ill-conditioning or collinearity problems and poor estimation of the model parameters [24]. It is therefore desirable to eliminate such redundancy from the data set by carefully selecting the key variables. Modeling tools such as multivariable statistical projection methods, which include partial least square (PLS), principal component regression (PCR) and regularized canonical correlation analysis (RCCA), address effectively handle this issue via regression on a smaller number of transformed variables called "latent variables". These modeling approaches result in well-conditioned parameter estimates and good model prediction [24]. Moreover, they were intensively studied and used for the rank reduction of the observed data as well as to reveal underlying relationships across variables. The data-based process monitoring approaches have broad applications [2], [25], [26]. One of them partial least square (PLS) method, has recently received much attention from statisticians and researchers [27]-[29]. PLS is a basic method of multivariate analysis and is a powerful tool in 
processes monitoring [2], [30], [31]. PLS also stands for projection on latent structures, determines an appropriate pair of latent variables (unmeasured variables), both input and output physical variables so that the transformed variables have the largest covariance [32]. Numerous extensions of PLS have been designed to meet various practical requirements. The multi-block PLS [33], modified PLS [26], dynamic PLS [25] and kernel PLS [34] are a few examples.

The occurrence of possible faults in process monitoring can be caused by malfunctioning sensors, also termed 'faulty sensors', or by irregular process modifications. The accurate detection of faults is crucial for maintaining a monitored system that meets all its designed requirements. However, conventional PLS-based $T^{2}$ and $Q$ charts are often ineffective or fail to detect faults as they make a decision based on the last observation alone. Thus, the main focus of our work is the development of more sensitive monitoring techniques that can detect faults in multivariate input-output data. To this end, we propose an effective fault detection approach based on continuous ranked probability score (CRPS) as an improved alternative for fault detection. CRPS has been used extensively in probabilistic forecasting as a metric for evaluating the quality of forecasts by quantifying the discrepancies between the observation (a scalar) and the cumulative distribution function (CDF) of the forecasting distribution [35], [36]. Our objective is to use CRPS for fault detection. Here, it is adopted as fault detection index. This method is attractive because it is intuitive and easy to implement. Additionally, it is important to note that other techniques based on the common distribution measures such as the Hellinger distance and the KullbackLeibler divergence [37], use residual distributions (before and after an anomaly) to detect potential faults. These distributionbased fault detection methods are batch. In other words, they need the entire a priori data to be available in order to compute residual distributions of training and testing data. In real processes, however, data are continuously collected, and in most cases, monitoring their key variables is needed online, as these variables are being measured. Therefore, it is essential to develop fault detection methods that handle online processes. The other CRPS compares a full distribution with a certain point which makes it very attractive for real-time fault detection. Furthermore, the closed form of the CRPS metric that exists for most classical parametric distributions, makes it more flexible for non-Gaussian data than for conventional charts such as the $T^{2}$, Q, MCUSUM and MEWMA charts, which are designed based on the assumption that the process observations are normally distributed. A CRPS metric is a powerful tool that has been used to quantify distances between probability distributions [35]. Additionally comparing each new observation with the full reference CDF, makes this chart very sensitive to significant any deviation in mean and/or variance of the monitored process. Therefore, it is very attractive for real-time fault detection. Until recently, CRPS metric, however, has not been used to improve the performance of univariate monitoring charts. Here, we focus on the development of a new CRPS monitoring technique based on latent variable regression models. Thus, integrating the feature-extraction capability of PLS modeling with change detection capacity of the CRPS scheme can provide very competitive performance in fault detection. We develop two new PLS-based CRPS monitoring techniques that can be very sensitive to small changes. The first approach uses the Shewhart scheme to evaluate the CRPS of the residuals from the PLS model. The second approach merges the CRPS into the exponentially weighted moving average monitoring chart. We apply the PLS-based CRPS approaches to simulated and real distillation column data, and compare its performances with the performance of conventional PLS-based $Q, T^{2}$, MEWMA and MCUSUM methods.

The following section briefly reviews the linear PLS method used for the multivariate process monitoring. Section III review the MEWMA and MCUSUM charts and how they can merged with PLS method and used for fault detection. We then introduce two new multivariate monitoring methods based on CRPS metric in Section IV. In Section V, we assess the efficiency of the developed approach using via a simulation study and a real data application, and Section VI concludes with a discussion.

\section{Partial Least Squares (PLS)-BASed Charts}

Consider a properly scaled input data matrix or measurement matrix $\mathbf{X} \in R^{n \times m}$ with $n$ measurements, $m$ input process variables and an output data matrix $\mathbf{Y} \in R^{n \times p}$ with $p$ output variables. PLS successively extracts latent variables (LVs) from both $\mathbf{X}$ and $\mathbf{Y}$ so that the covariance between the extracted LVs is maximized. More details on PLS modeling are included in [17], [29]-[31], [38]. A PLS model comprises itself two models: the inner and outer models [32], [38] (see Figure 1). The outer model establishes the relationships between the input and output matrices with their LVs [38]:

$$
\left\{\begin{array}{l}
\mathbf{X}=\widehat{\mathbf{X}}+\mathbf{E}=\sum_{i=1}^{l} \mathbf{t}_{i} \mathbf{P}_{i}^{T}+\mathbf{E}=\mathbf{T} \mathbf{P}^{T}+\mathbf{E} \\
\mathbf{Y}=\widehat{\mathbf{Y}}+\mathbf{F}=\sum_{i=1}^{l} \mathbf{u}_{i} \mathbf{q}_{i}^{T}+\mathbf{F}=\mathbf{U} \mathbf{Q}^{T}+\mathbf{F}
\end{array}\right.
$$

where $\widehat{\mathbf{X}}$ and $\widehat{\mathbf{Y}}$ contains the most significant variations present in data $\mathbf{X}$ and $\mathbf{Y}$ respectively, $\mathbf{T} \in R^{n \times l}$ and $\mathbf{U} \in R^{n \times q}$ represent a matrix of the transformed uncorrelated variables, and $l$ is number of retained LVs. Here, $l$ is such that there is no important process information left in $\mathbf{E} \in R^{n \times m}$ and $\mathbf{F} \in R^{n \times p}$; $\mathbf{E}$ and $\mathbf{F}$ are expected to contain only the random noise. The matrices $\mathbf{P} \in R^{m \times l}$ and $\mathbf{Q} \in R^{p \times q}$ are the loading of $\mathbf{X}$ and $\mathbf{Y}$, respectively. Figure 1 shows a schematic example of the basic principle PLS in which $m=3, p=3$, and $l=1$. In PLS, it is very crucial to find the optimal number of LVs to be kept in the model [39]. In this paper, the cross-validation technique is employed to select the number of retained LVS [39]. As per this method, the total fault-free data set is split into two sets (i.e., training and testing data set). The PLS model is developed using the training data set, and then tested with the testing data set. The prediction ability of the developed model is determined using mean square error (MSE) based on testing data set. As the latent variable is added iteratively to 
the model, MSEs are calculated using the testing data set. The optimal number of latent variables is determined by locating the minimum of MSE. The latent variables of input and output data are related via the inner model as:

$$
\mathbf{U}=\mathbf{T B}+\mathbf{H},
$$

where $\mathbf{B}$ and $\mathbf{H}$ are the regression matrix and the residual matrix, respectively. The regression coefficients of the regression matrix $\mathbf{B}$ are determined by minimizing the residuals $\mathbf{H}$. We can compute $\mathbf{Y}$ as

$$
\mathbf{Y}=\mathbf{T B} \mathbf{Q}^{T}+\mathbf{F}^{*}
$$

where matrix $\mathbf{F}^{*}$ is the residual matrix presenting the unexplained variance. The extraction of each pair of LVs, $t_{j}$ and $u_{j}$ $(j=1, l)$ is done iteratively via the non-linear iterative partial least squares (NIPALS) algorithm [30].

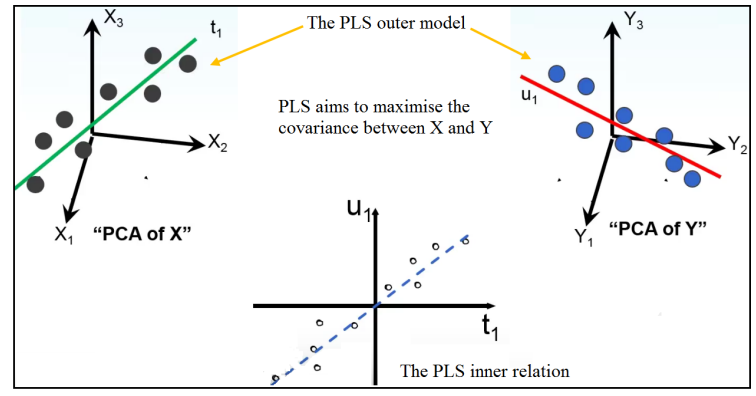

Fig. 1: Principle of PLS.

Once the PLS model is constructed, residuals are used in the fault detection step by control schemes, such as Hotelling $T^{2}$ and square prediction error (SPE) charts [40]. The $T^{2}$ statistic monitors changes in the LVs subspace. The SPE statistic, also termed $Q$, monitors residual subspace.

$$
T^{2}=\sum_{i=1}^{l} \frac{t_{i}^{2}}{\sigma_{i}^{2}},
$$

where, $\sigma_{i}^{2}$ is the estimated variance of the corresponding LV $t_{i}$. $T^{2}$ quantifies changes in LV's subspace. An abnormal variation in the LV's subspace is declared if some points overpass the statistic threshold, $T_{\alpha}^{2}$ [31],

$$
T_{i}^{2}>\chi_{1-\alpha, l}^{2} \text {. }
$$

The $Q$ statistic is calculated as in [31]:

$$
Q=\mathbf{e}^{T} \mathbf{e}
$$

It is designed to monitor the prediction error. $\mathbf{e}=\mathbf{x}-\hat{\mathbf{x}}$ represents the residual, which is the difference between the measured variable, $\mathbf{x}$, and the predicted variables, $\hat{\mathbf{x}}$, using the PLS model. The SPE quantifies the deviation between each sampling data and the model. The SPE control limits are given in [41].

It is designed to monitor the prediction error. The SPE quantifies the deviation between each sampling data and the model. We claim that there is an anomaly in the monitored process when

$$
Q>Q_{\alpha},
$$

where $Q_{\alpha}$ is a decision threshold, defined by [31]

$$
Q_{\alpha}=\varphi_{1}\left[\frac{h_{0} c_{\alpha} \sqrt{2 \varphi_{2}}}{\varphi_{1}}+1+\frac{\varphi_{2} h_{0}\left(h_{0}-1\right)}{\varphi_{1}^{2}}\right],
$$

where $\varphi_{i}=\sum_{j=l+1}^{m} \lambda_{j}^{i}$, for $i=1,2,3, h_{0}=1-\frac{2 \varphi_{1} \varphi_{3}}{3 \varphi_{2}^{2}}$, and $c_{\alpha}$ is the confidence limits for the $1-\alpha$ percentile in a normal distribution. As shown previously, control limits obtained from $Q$ and $T^{2}$ statistics require the assumption that the observed values are temporally non-correlated and normally distributed. PLS- $T^{2}$ and PLS- $Q$ approaches fail to detect changes [42]. Motivated by the sensitivities of MEWMA and MCUSUM to small faults [42] and the powerful of the CRPS metric to quantify a distance between a certain point and a probability distribution [36], we propose three innovative monitoring charts to effectively monitor the mean in multivariate processes: PLS-based MEWMA, MCUSUM and CRPS fault detection methods. In Section III, below, we briefly describe how these fault detection methods are designed.

\section{PLS-BASED MCUSUM AND MEWMA CHARTS}

MCUSUM and MEWMA schemes, which are based on a decision rule that account for information from past observations, are used as a better alternative to conventional PLS approach (i.e., $T^{2}$ and $Q$ ), particularly for detecting small faults [42]. This section briefly describes the MEWMA and MCUSUM charts.

\section{A. MCUSUM monitoring chart}

MCUSUM schemes have the ability to detect changes in the process mean [42]. The MCUSUM chart aggregates all the information from past and current samples in the decision procedure. Numerous versions of the MCUSUM monitoring scheme are reported in the literature [42]. We use the verson proposed by Crosier [42] that receives much attention in the literature. Let $\mathbf{X}_{t}=\left(X_{1}, X_{2}, \ldots, X_{m}\right)^{T}$, be a sequence of i.i.d. $\mathscr{N}_{p}(\mu, \Sigma)$ random vectors obtained from a $p$-dimensional production process. The process is under nominal conditions when $\mu=\mu_{0}$, and under abnormal operating conditions when $\mu \neq \mu_{0}$, where $\mu_{0}$ is the mean of fault-free data. The decision statistic of the MCUSUM scheme, $C_{t}$, can be computed by the recursive formulas [42].

$$
C_{t}=\sqrt{L_{t}^{T} \Sigma^{-1} L_{t}}
$$

where

$L_{t}=\left\{\begin{array}{ll}0 & \text { if } C_{t} \leq k \\ \left(L_{t-1}+X_{t}-\mu_{0}\right)\left(1-\frac{k}{C_{t}}\right) & \text { else }\end{array}\right.$,

and $\quad C_{t}=\sqrt{\left.\left(L_{t-1}+X_{t}-\mu_{0}\right)^{T}\right) \Sigma^{-1}\left(L_{t-1}+X_{t}-\mu_{0}\right)}$, where $\mu_{1}$ represents the out-of-control process mean vectors. Crosier [42] recommended that $L_{0}=0$ and $k=\frac{\sqrt{\left(\mu_{1}-\mu_{0}\right)^{T} \Sigma^{-1}\left(\mu_{1}-\mu_{0}\right)}}{2}$ and follow the convention of resetting the MCUSUM scheme following a signal. This chart gives a signal of mean shift when $C_{t}$ is larger than the critical value $H$, where $H$ is a decision threshold chosen to achieve a pre-defined probability of false alarm [42]. The $H$ value can be determined by a Monte Carlo simulation [42]. 


\section{B. MEWMA monitoring chart}

Let us consider the input data matrix $\mathbf{X}_{t}=\left(X_{1}, X_{2}, \ldots, X_{p}\right)^{T}$, where $X_{i} \in \mathbb{R}^{m}$. Herein, $\mathrm{m}$ represents the number of the process variables, that are computed at each time point. The MEWMA statistic is computed at each observation time point as follows [42]:

$$
\mathbf{Z}_{t}=\Lambda \mathbf{X}_{t}+\left(\mathbf{I}_{m \times m}-\Lambda\right) \mathbf{Z}_{t-1},
$$

where the initial value $\mathbf{Z}_{0}=\left(\mu_{X_{1}}, \mu_{X_{2}}, \ldots, \mu_{X_{m}}\right)^{T} \in \mathbb{R}^{m}$ is generally set equal to the sample mean of the historical data without change (training data). $\Lambda=\operatorname{diag}\left(\lambda_{1}, \lambda_{2}, \ldots, \lambda_{m}\right)$ is the diagonal $m \times m$ matrix of the smoothing constant, with $\lambda_{j} \in[0,1]$, and $\mathbf{I}_{m \times m}$ denotes the identity matrix. Generally, a small value of $\lambda$ is selected (i.e., more weight is given to past observations) to detect small deviations, whereas a large $\lambda$ value is suitable to detect large changes [42]. The charting statistic plotted in the chart is:

$$
\mathbf{V}_{t}^{2}=\mathbf{Z}_{t}^{T} \Sigma_{\mathbf{Z}_{t}}^{-1} \mathbf{Z}_{t},
$$

where $\Sigma_{\mathbf{Z}_{t}} \in \mathbb{R}^{m \times m}$ is the covariance matrix of $\mathbf{Z}_{t}$ [42],

$$
\Sigma_{Z_{t}}=\frac{\lambda}{2-\lambda}\left[1-(1-\lambda)^{2 t}\right] \Sigma,
$$

where $\Sigma$ is the covariance of the input data. The MEWMA chart with the charting statistic $\mathbf{V}_{t}^{2}$ gives a signal of mean shift when $\mathbf{V}_{t}^{2}$ overpass a decision threshold $h$. The threshold $h$ can be determined via simulation for a given probability of false alarm [42].

Combining the benefits of PLS modeling with those of the memory monitoring charts, MCUSUM and MEWMA, should result in an enhanced fault detection system, in particular for detecting small faults. To achieve this coupled approach, we develop the PLS-based MCUSUM and MEWMA fault detection schemes. We apply MCUSUM and MEWMA charts to ignored LVs obtained from the PLS model. The ignored latent variables associated with small eigenvalues should be useful for detecting small shifts. The ignored latent variables, which capture the variability that arises from noise, represent the residuals of the process. Indeed, the ignored LVs are close to zero under normal operation and change significantly in the presence of faults. Here, the ignored LVs are used as faults indicator. Another option for enhancing the sensitivity of the PLS-based monitoring approach, with respect to small changes, is to use the CRPS metric. In the next section, we present the design of the PLS-CRPS approach.

\section{PLS-BASED CRPS CHART}

In this section, we develop an innovative fault detection approach using the continuous ranked probability score (CRPS). The CRPS metric has been extensively used to assess the forecasting accuracy of the probabilistic forecasting field [35], [36], [43]. By using CRPS, both sharpness and accuracy of forecasting are taken into account in evaluating the quality of forecasts [44].

Definition 1 (Continuous ranked probability score). let us consider $X$ be a random variable with the cumulative distribution function $(C D F) F$, with $F(y)=\mathbb{P}[X \leq y]$. Let $x$ be the observation, and $F$ the CDF associated with an empirical probabilistic forecast. Let $\mathbb{1}\{$.$\} represents the indicative$ function (if $x<y$, the indicative function value is 1 ; and 0 otherwise). The CRPS between $x$ and $F$, which measures the integral square difference between the CDF of the prediction $F$, and the corresponding CDF of the observed variable, is defined as [43]:

$$
\operatorname{CRPS}(F, x)=\int_{-\infty}^{\infty}(F(y)-\mathbb{1}\{y \geq x\})^{2} d y .
$$

The schematic representation of CRPS is presented in Figure 2. The CRPS is the area between the two CDFs, $F$ and $\mathbb{1}\{x\}$ (the shaded area). A perfect CRPS score is zero. Let us

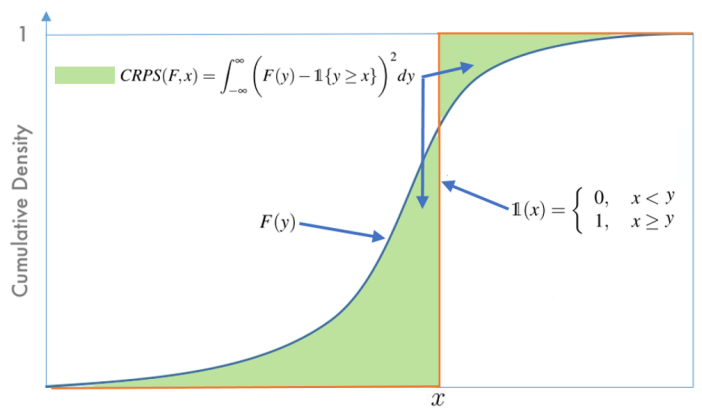

Fig. 2: An illustration of CRPS (colored area).

consider the particular case when the predictive distribution is a Gaussian vector with mean $\mu$ and variance $\sigma^{2}$, the closed form of CRPS is [45],

$$
\begin{aligned}
\operatorname{CRPS}\left(\mathscr{N}\left(\mu, \sigma^{2}\right), x\right)= & \sigma\left[\frac{x-\sigma}{\sigma}\left(2 \Phi\left(\frac{x-\sigma}{\sigma}\right)-1\right)\right. \\
& \left.+2 \phi\left(\frac{x-\sigma}{\sigma}\right)-\frac{1}{\sqrt{\pi}}\right]
\end{aligned}
$$

where $\phi$ and $\Phi$ represent the standard Gaussian PDF and $\mathrm{CDF}$, respectively. Analytic forms of $\mathrm{CRPS}(\mathrm{F}, \mathrm{y})$ exist for most classical parametric distributions such as the Laplace distribution, Gamma distribution, Log-normal distribution, Exponential distribution, and Beta distribution [46]-[48]. In many applications, the closed form of $F$ is not available and must be estimated from the data sets.

Here, we introduce the CRPS metric for process monitoring and propose an effective anomaly detection approach to monitor multivariate input-output processes. This approach merges a PLS model and a CRPS-based chart to separate normal from abnormal features by simultaneously taking advantage of the feature-extraction capability of the PLS model and the sensitivity capacity to anomalies of CRPS. We first build an appropriate PLS under fault-free conditions. This model is then applied to inspect process variables for abnormal events by looking for future deviations from normality. In this approach, the output PLS residuals, which are defined as the differences between the measured response variables and those estimated by the PLS model, are used as anomaly indicators. The CRPS is used to quantify the distance between a reference CDF of the output residual, which is obtained from the PLS model using fault-free data, and the actual residual observation. CRPS is suitable for real-time detection; each incoming single-value 
observation is immediately compared to the CDF of fault-free residual. Indeed, under nominal conditions, CRPS becomes closer to zero, whereas a larger CRPS value is obtained under abnormal conditions that lead to poor performance. To check whether the process is running under control at the i-th time point, it is natural to consider the following hypotheses: the null hypothesis $\left(\mathscr{H}_{0}: \operatorname{CRPS}(F, x) \leq h\right)$ and the alternative hypothesis $\left(\mathscr{H}_{1}: \operatorname{CRPS}(F, x)>h\right)$. Hence, to detect faults statistically, two fault detection schemes based on the CRPS measurements and Shewhart and EWMA schemes have been introduced. The first scheme is the CRPS-Shewhart monitoring scheme, which applies Shewhart chart to the CRPS sequences to detect faults. The second scheme merges CRPS and EWMA chart, where the EWMA chart is applied to the CRPS sequences for fault detection.

\section{A. Mixed CRPS-Shewhart monitoring scheme}

The mixed CRPS-Shewhart chart combines CRPS measure and Shewhart chart also know as the three-sigma rule. Specifically, in CRPS-Shewhart chart, CRPS measurements are used as the input to Shewhart chart for anomaly detection. The three-sigma rule is used to compute the detection threshold $h$, expressed as:

$$
h=\mu_{0}^{C R P S}+3 \sigma_{0}^{C R P S},
$$

where $\mu_{0}^{C R P S}$ and $\sigma_{0}^{C R P S}$ are the mean and standard deviation of CRPS measurements under anomaly-free case. A fault is detected when some points exceed the decision threshold of the CRPS-based chart, $h$. Next, we describe the CRPS-EWMA scheme.

\section{B. Mixed CRPS-EWMA monitoring scheme}

Here, we briefly describe the proposed PLS-based CRPSEWMA fault detection scheme. PLS residual sequences have been utilized as a fault detection indicator. Fault decision statistic has been designed using CRPS as a measure between PDFs of fault-free residuals and actual residual then mixed with EWMA chart for fault detection. The main advantage of combining CRPS and EWMA for fault detection is that EWMA scheme aggregates all of the information from past and actual samples in the decision rule. Defining the vector of CRPS measurements as computed in (14): CRPS = $\left[d_{1}, \ldots, d_{j}, \ldots, d_{n}\right]$. The CRPS-EWMA fault decision statistic is computed as follows:

$$
z_{t}^{C R P S}=v d_{t}+(1-v) z_{t-1}^{C R P S},
$$

where the initial value, $z_{0}^{C R P S}$ is the anomaly-free mean of CRPS vector, $\mu_{0}^{C R P S} . v(0<v \leq 1)$ is a smoothing parameter. Generally, a small value of $v$ is selected (i.e., more weight is given to past observations) to detect small changes in the process mean. A value of $v$ is usually chosen between 0.2 and 0.3 [49]. When the CRPS-EWMA statistic, $z_{t}^{C R P S}$, is beyond the decision threshold, $h_{E W M A}$, then we claim that there is a fault. Otherwise, the supervised process is considered in control. The CRPS-EWMA fault detection threshold is designed as [50],

$$
h_{E W M A}=\mu_{0}^{C R P S}+L \sigma_{0}^{C R P S} \sqrt{\left(\frac{v}{(2-v)}\left[1-(1-v)^{2 t}\right]\right.},
$$

where $L$ is the width of the control limits. When a univariate EWMA chart is utilized to detect abnormal measurements in a single variable, then the univariate EWMA chart usually has two parameters involved (i.e., $v$ and $L$ ).

The proposed strategy comprises two main stages: model construction based on fault-free dataset, and online fault detection using the CRPS-based monitoring chart. In model identification, our objective is to find a sound PLS model for estimating the LV space or its complementary part, the residual space (in fault detection problem, the later is critical). The schematic representation of the proposed PLS-CRPS algorithm is presented in Figure 3.

\section{EXPERIMENTAL RESULTS AND DISCUSSION}

This section reports on the effectiveness of the proposed PLS-CRPS approach through simulation and practical distillation column data. We compared the proposed PLS-CRPS algorithm with the PLS-based $T^{2}, Q$, MCUSUM, and MEWMA algorithms.

\section{A. Monitoring of distillation Column Data}

To validate the performance of the PLS-CRPS approach, we used data collected from a distillation column simulated via Aspen (see [51] for details). The generated data consist of twelve input variables (i.e., ten temperatures $\left(T_{c}\right)$ collected at different stages in the monitored column, the feed flow rates, and the reflux stream), and one output variable (i.e., the composition of the light component in the distillate stream). A data set consisting of 1024 samples is generated using an Aspen simulator. Figure 4 illustrates the dynamic inputoutput data of the distillation column under nominal operating conditions with a signal-to-noise ratio of 10 . The first half of the data set is used to train the PLS model and the rest of the data is used for testing. Cross-validation is used to choose the number of LVs in the PLS model. Three LVs are selected in order to build the PLS model (provided $R^{2}=$ 0.96 and RMSE $=0.003$ ). $R^{2}$ values above 0.9 and a low RMSE value of 0.003 demonstrates that PLS provides good predictive quality. Scatter plots of measured values against estimated values (from the PLS model) are presented in Figure 5. Results demonstrate that the constructed PLS model performs well when describing distillation data. When the model is used for prediction, the mean and standard deviations of training data are used to scale the available testing data. In Table I, we introduce two types of faults in the testing datasets and compare the performances of the six charts. In each experiment, we measure the false alarm rate (FAR) and the miss detection rate (MDR). The smaller the FAR and MDR are, the better the detection rate is [4].

\section{Case (i) - Bias sensor fault:}

In this section, we assess the performance of the PLSCRPS algorithm for detecting abnormal abrupt increases in temperature measurement, $T_{C 3}$, for sampling times ranging from 100 to 150 . This change is equal to $2 \%$ of the total variation found in the raw data. Monitoring results of the $Q$ and 


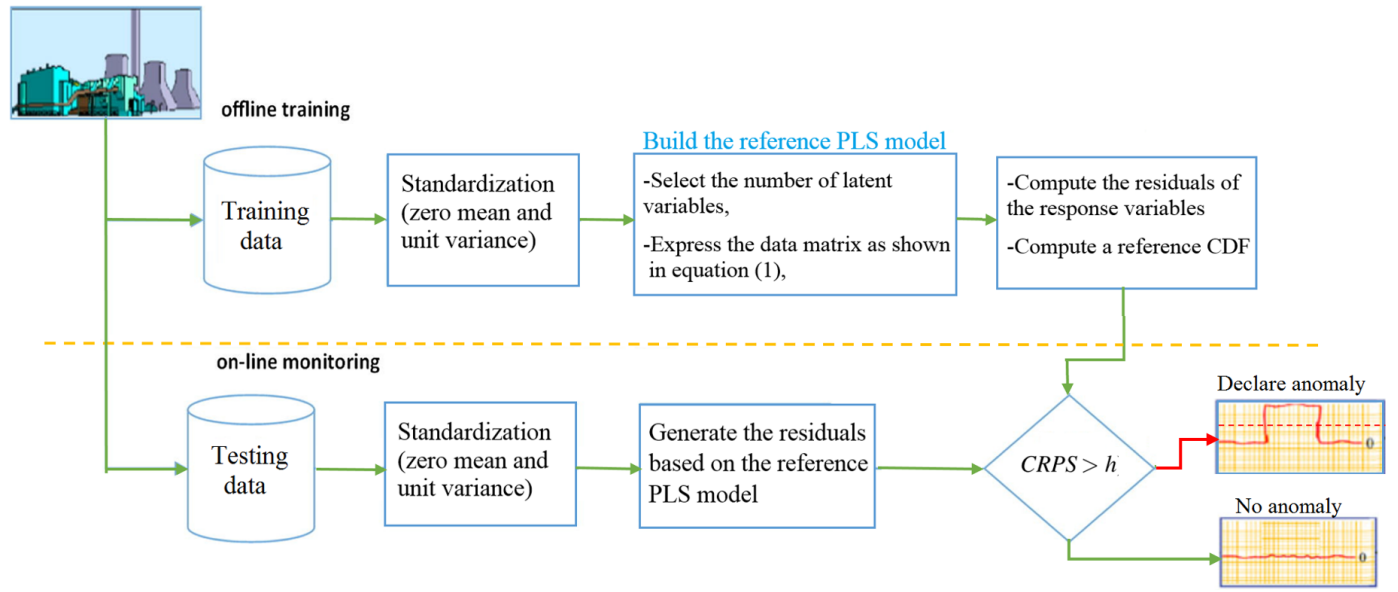

Fig. 3: Flowchart of PLS-CRPS monitoring scheme.
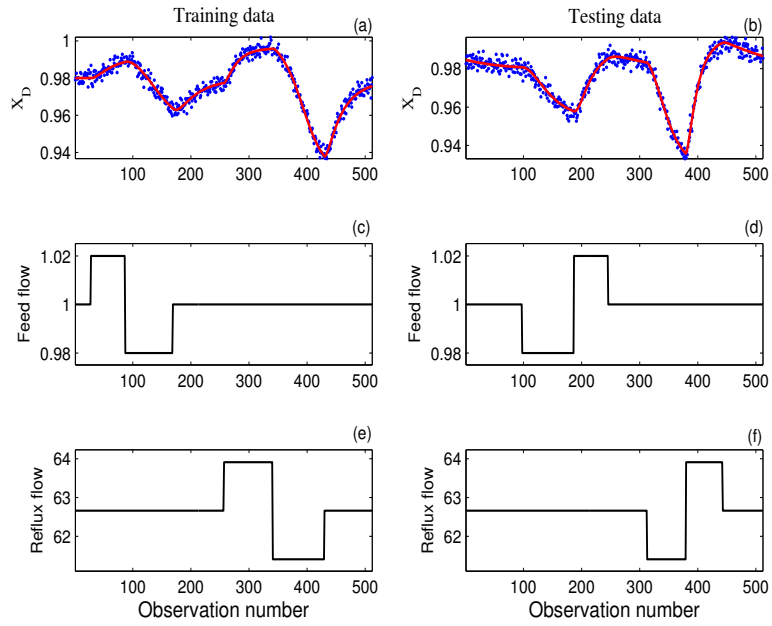

Fig. 4: Dynamic Input-output data (solid red line represent PLS model prediction; blue dots represents the measured data).

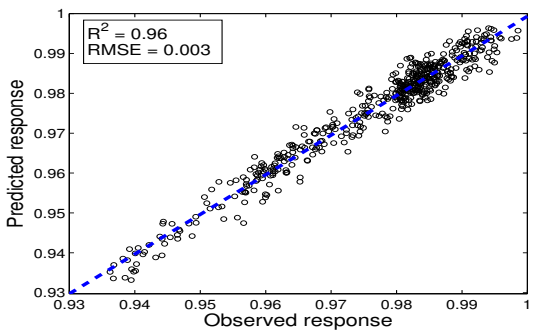

Fig. 5: Measured versus predicted values from the fitted PLS model.

TABLE I: Fault types introduced to the testing dataset.

\begin{tabular}{cll} 
Fault Type & Model & $\begin{array}{l}\text { Parameters } \\
\text { Bias Fault }\end{array}$ \\
& $T c_{3}(t)=T c_{3}(t)+b \cdot$ & $b:$ shift in temperature \\
& & $T c_{3}$ for $t \in \mathscr{I}$ \\
\hline Drift Fault & $T c_{3}(t)=T c_{3}(t)+s t_{0} \cdot$ & $s:$ slope of the drift \\
& & for the starting point $t_{0}$ \\
\hline
\end{tabular}

$T^{2}$ charts are shown in Figure 6(a)-(b). This figure shows that neither of these charts can produce a signal. In this case study, the PLS-based $Q$ and $T^{2}$ charts are insensitive to the simulated faults because they are designed to detect moderate and large mean shifts, which is not the case here as the simulated mean shift is quite small. Figure 6(c), shows detection results of the PLS-MCUSUM algorithm with $(k=0.5$ and $H=6.88)$. We see that the PLS-CUSUM chart detects this fault, but that it also results in a high false alarm rate (i.e., $\mathrm{FAR}=59.95 \%$ ) and some missed detections (MDR $=4 \%$ ). However, the results of the PLS-MEWMA chart with $\lambda=0.25$, represented in Figure 6(d), clearly show the capacity of this approach to detect this small anomaly with zero false alarm (i.e., $F A R=0 \%$ ) and low missed detections (MDR=2\%). The results from the PLSbased CRPS-Shewhart algorithm are shown in Figure 6(e). From the plot, we see that the simulated fault is correctly detected by the chart based on CRPS-Shewhart (i.e., FAR= $0.2165 \%$ and $\mathrm{MDR}=0 \%$ ). The monitoring results from the CRPS-EWMA approach are presented in Figure 6(f). In this example, the CRPS-EWMA detected this bias fault without false alarms (i.e., $\mathrm{FAR}=0 \%$ ) and resulted in $\mathrm{MDR}=0.21 \%$.

\section{Case (ii) - Intermittent sensor fault:}

Here, we assess the PLS- CRPS approach in presence of intermittent faults. Intermittent faults occur and disappear repeatedly. First, we inject a bias of amplitude $2 \%$ of the total variation of raw data in temperature $T c_{3}$ for samples 50 to100, and for samples 150 to 250 . Detection results of the six charts are given in Figure 7(a)-(d). Conventional PLS-based charts (i.e., $T^{2}$ and $Q$ statistics) are not sensitive to process mean faults when these faults are small (see Figure 7(a) and 12(b)). In this example, the $T^{2}$ resulted in FAR $=7.75 \%$ and $\mathrm{MDR}=100 \%$, and the $Q$ resulted in $\mathrm{FAR}=4.75 \%$ and MDR $=100 \%$. The PLS-MCUSUM chart (Figure 7(c)) detects this type of fault, but with several false alarms and some missed detections (i.e., $\mathrm{MDR}=1.33 \%$ and $\mathrm{FAR}=86.19 \%$ ). Figure 7(d) shows that the PLS-MEWMA chart correctly detects these intermittent faults without false alarms, (i.e., $\mathrm{MDR}=1.33$ and $\mathrm{FAR}=0 \%$ ). The plot in Figure 7(e) clearly show the capability of the PLS-based CRPS-Shewhart chart in detecting this intermittent faults. The PLS-CRPS chart 

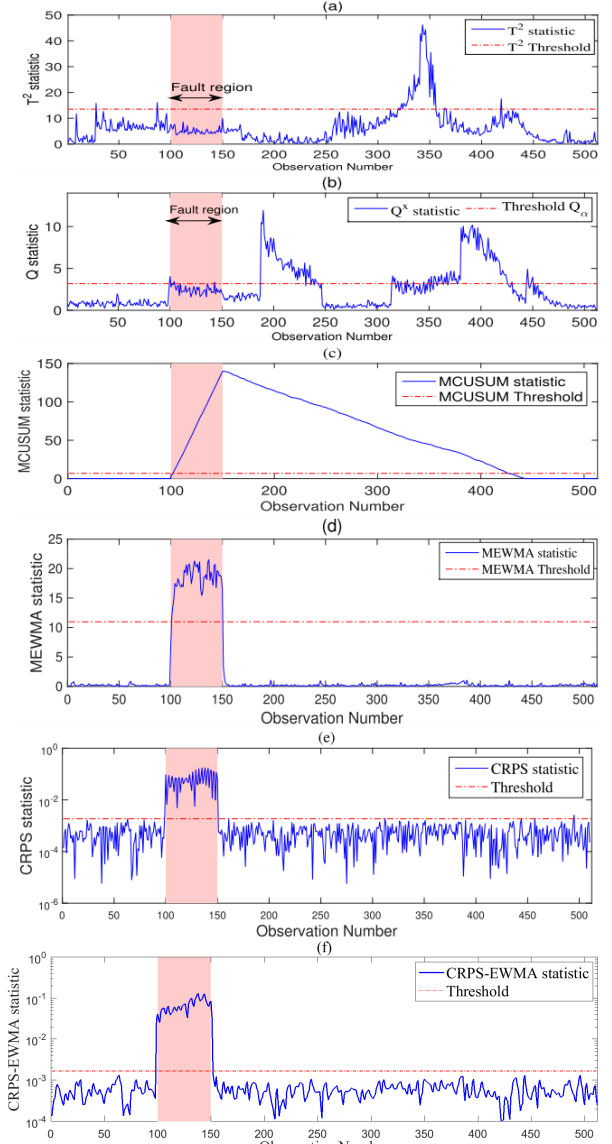

Fig. 6: Monitoring results of the $T^{2}$ (a), $Q$ (b), MCUSUM (with $k=0.5$ and $H=6.88$ ) (c), MEWMA with $\lambda=0.3$ (d), CRPS-Shewhart (e) and CRPS-EWMA (with $v=0.3$ ) (f) charts in the presence of bias fault in $\mathbf{x}_{5}$ from sample 100 to 150 (case (i)).

performs reasonably well (i.e., $\mathrm{MDR}=0.66$ and $\mathrm{FAR}=1.2 \%$ ). Figure 7(f), shows detection results of the CRPS-EWMA algorithm with $(v=0.25$ and $L=3)$. We see that the CRPS-EWMA chart correctly detects the introduced faults without missed detection and results in few false alarms (i.e., $\mathrm{FAR}=1.14 \%$ and $\mathrm{MDR}=0 \%$ ). These results show again the superiority of the CRPS-EWMA approach over other charts. Figure 7(f) shows detection results of the CRPS-EWMA algorithm. We see that the CRPS-EWMA chart correctly detects the introduced faults without missed detection and results in few false alarms (i.e., FAR $=1.14 \%$ and $\mathrm{MDR}=$ $0 \%)$. This example testifies again the powerful of the proposed charts in detecting small faults (see Figures 7(e)-(f)). The CRPS-EWMA chart is good for detecting relatively small and persistent faults, its performance is slightly higher to that of the MEWMA chart, and it is easy to understand and implement.

\section{Case (iii): Drift sensor fault:}

Gradual fault usually characterized by a slow increase of abnormal measurements. In this case, we evaluate that there is a possibility that PLS-CRPS may detect gradual faults. We observe a drift with a slope of 0.01 , compared to raw measurements of $T_{C 3}$ from sample number 250. Results for
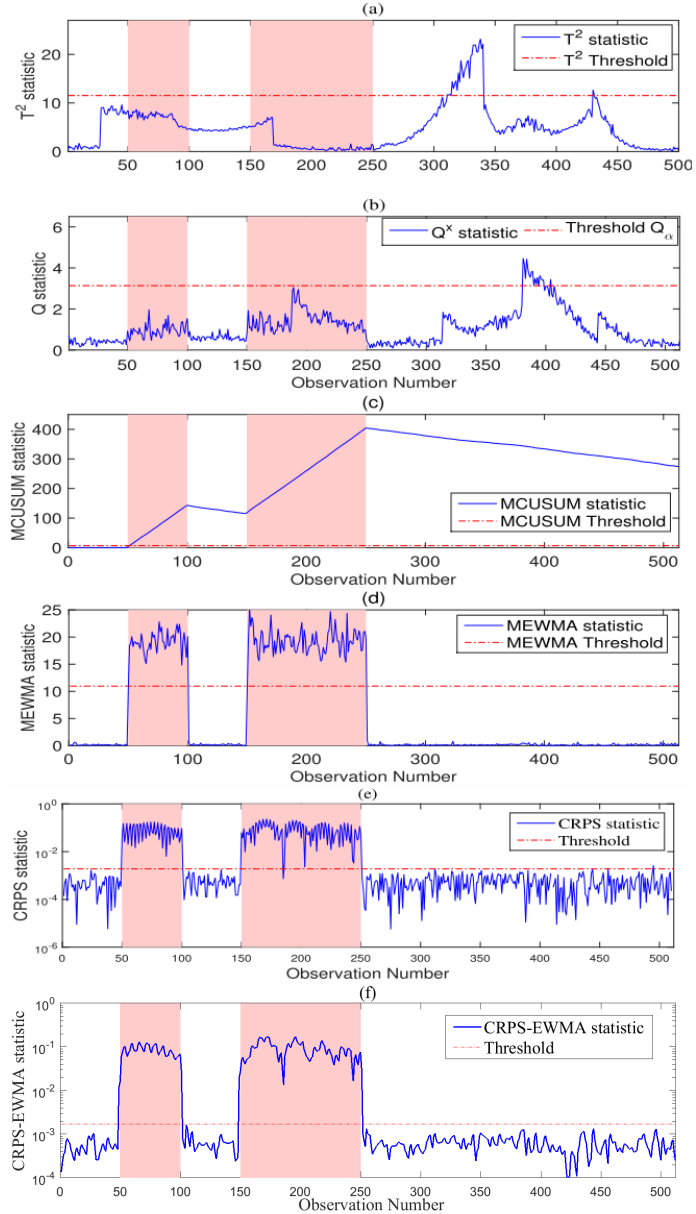

Fig. 7: Monitoring results of the $T^{2}$ (a), $Q$ (b), MCUSUM (with $k=0.5$ and $H=6.88$ ) (c), MEWMA (with $\lambda=0.3$ )(d), CRPS-Shewhart (e) and CRPS-EWMA (with $v=0.3$ ) (f) charts in the presence of intermittent faults in $x_{5}$ (case (ii)).

$T^{2}$ and $Q$ charts are presented in Figures 8(a)-(b). Figure 8(a) shows that the $T^{2}$ statistic is not able to detect the introduced change. Figure 8(b) indicates that the PLS- $Q$ scheme detects an anomaly for sample 356 . The PLS-based MCUSUM and MEWMA charts gives the first signal of fault at the 330th time point (see Figures 8(c)-(d)). In the MCUSUM chart, $k$ and $h$ are chosen to be 0.5 and 6.88 , respectively; in the MEWMA chart, $\lambda$ is chosen to be 0.5 . The monitoring results based on the PLS-based CRPS-Shewhart approach are presented in Figure 8(e). A fault is first detected at the 290th time point. Figure 8(f) indicates that CRPS-EWMA scheme detected an anomaly at sample 273. Therefore, fewer extra observations are required for the CRPS-EWMA scheme to detect this fault, compared to the other schemes. As a summary, this example demonstrates that the convention PLS-based $T^{2}, Q$, MCUSUM and MEWMA charts cannot be effective in detecting small faults, but the performance of the proposed charts are reasonably satisfactory. The PLS-CRPS chart performs the best in this case.

\section{B. Experimental Study Using Packed Bed Distillation Column}

We perform experimental investigations to test the effectiveness of the PLS-CPRS approach using a packed bed distillation 

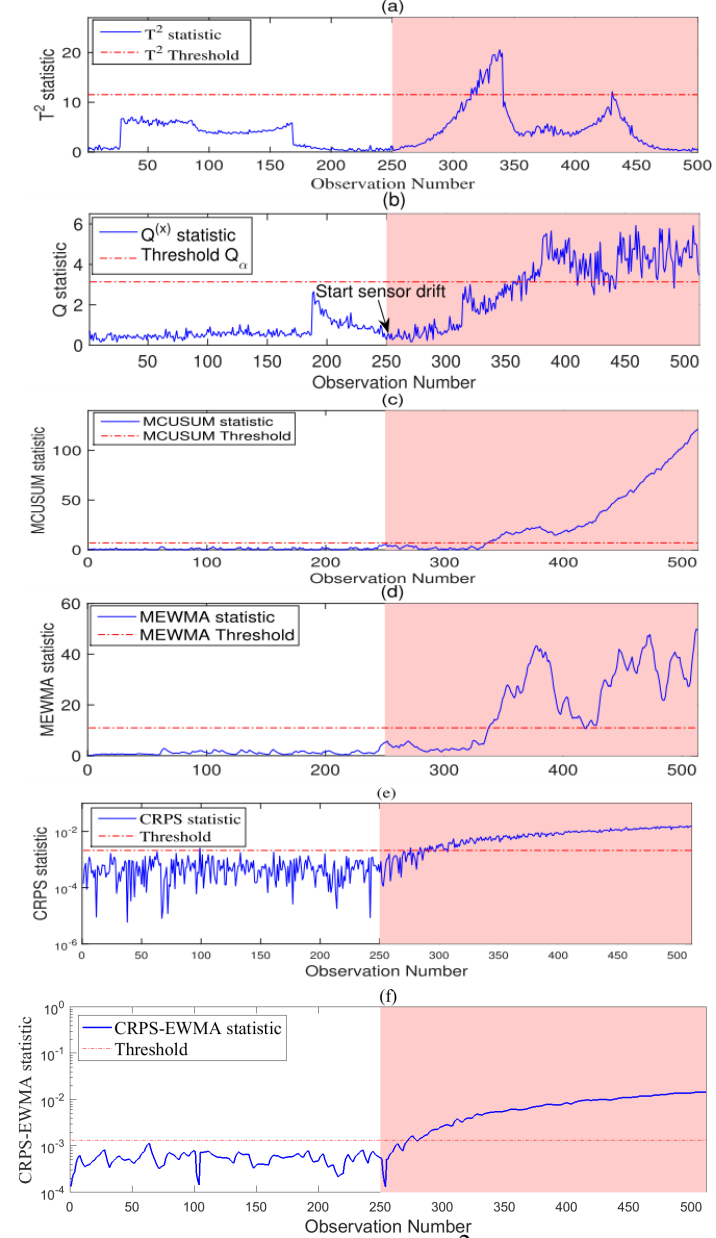

Fig. 8: Monitoring results of the $T^{2}$ (a), $Q$ (b), (with $k=$ 0.5 and $H=6.88$ ) (c) MEWMA (with $\lambda=0.3$ ) (d), CRPSShewhart (e), CRPS-EWMA (with $v=0.3$ ) (f) statistics in the presence of drift fault with slope 0.01 in temperature sensor $\mathbf{x}_{5}$ from sample 250 (case (iii)).

column (Figure 9). This column is made of stainless steel and is six inches in diameter. It consists of three sections, 20-feet high, packed with Koch Sulzer material up to the top. A liquid distributor is fixed at the top of each section to ensure a uniform liquid distribution. The column has two feed points at different locations on the column: one above the bottom packed bed, and one above the middle -packed bed. The system is designed so that the feed and reflux flows can be regulated using controlled pumps, i.e, FIC 401 (for the feed flow) and FIC 403 (for the reflux flow) (Figure 9). To monitor the temperature profile of the column, six temperature sensors (Resistance Temperature Detectors i.e., T1 408 to T1 416 in Figure 9) are installed at different locations on the column. In addition, a RTD sensor, a flow sensor, and a level sensor are used at different locations just to monitor other parameters of the system for a smooth conduct of experiment. A mixture of methanol and water is used in the experimental study. Column control is ensured by the Delta V system, an industrial quality Distributed Control System (DCS), Delta V from Emerson Process Management.
1) Data Collection: The study first involves the generation of perturbation data of the packed bed distillation column. The resulting datasets are used for PLS model estimation and validation purposes. The feed and reflux flow rates are perturbed approximately at the nominal operating conditions described in [51]. The variables are sampled constant sampling rated at $4 \mathrm{sec}(\mathrm{T})$. The perturbation in the inputs is carried out sequentially. First, the feed flow is perturbed at $\pm 50 \%$, and the system is allowed to reach nominal conditions. The reflux flow is then perturbed around nominal conditions, i.e. $\pm 40 \%$. Figure 10 shows the perturbations in the inputs and Figure 12 shows the variations of the column composition when changing the input variables.

For the modeling part, a total of six temperatures, flow rates of feed and reflux are considered as input variables. The composition of the light component (methanol) in the distillate (i.e., $\mathrm{xD}$ ) considered as an output variable. Figure 12 shows the measured $\mathrm{xD}$ and the predicted $\mathrm{xD}$ obtained by the reference PLS model.

After the process model has been successfully constructed using training data, we can proceed with fault diagnosis. Two different types of faults are simulated to assess the detection ability of the proposed algorithms: abrupt anomalies and gradual faults.

\section{Case (a) - Abrupt fault:}

524 samples are collected from the distillation column (Figure 9) for testing purposes. An abrupt change is simulated by introducing a small constant change of 5\% magnitude to the temperature measurement, $T_{3}$, between sample times 200 and 300. As expected, the PLS- $T^{2}$ chart is shown in Figure 13(a). The PLS- $T^{2}$ is ineffective when the magnitude of the fault is relatively small (see Figure13(a)). Figure 13 (b) shows that the fault is detected by the $Q$ chart but with a high rate of false alarms (FAR $=77.46 \%$ ). The performance of the PLS-based MCUSUM and MEWMA charts are shown in Figure 13(c)-(d). Using the PLS-MCUSUM method, the FAR and MDR are found to be $2 \%$ and $28 \%$, respectively. The PLS-MEWMA method leads to a lower FAR and MDR of $0.7 \%$ and $4 \%$, respectively. The PLS-CRPS scheme applied to the testing dataset is represented in Figure 13(e). This figure shows that the PLS-CRPS scheme detects the fault, but with some missed detections (MDR=14\%). Figure 13(f) shows that the CRPS-EWMA scheme detects this introduced fault but with few false alarms (i.e.,FAR $=0.73 \%$ and $\mathrm{MDR}=0 \%$ ). Anomaly detection based on CRPS-EWMA scheme improves the detection quality by reducing the rate of false alarms and missed detections. These results show the superiority of the CRPS-EWMA approach over other approaches.

\section{Case (b) - Drift sensor fault:}

If faults are left undetected for a long period of time serious damages might occur. Much time and money can be saved by early detection of faults. For the experiment using a gradual fault, a slope of 0.05 is introduced to the temperature sensor, $T C_{3}$, starting at sample 200 and finishing with sample 524, the 


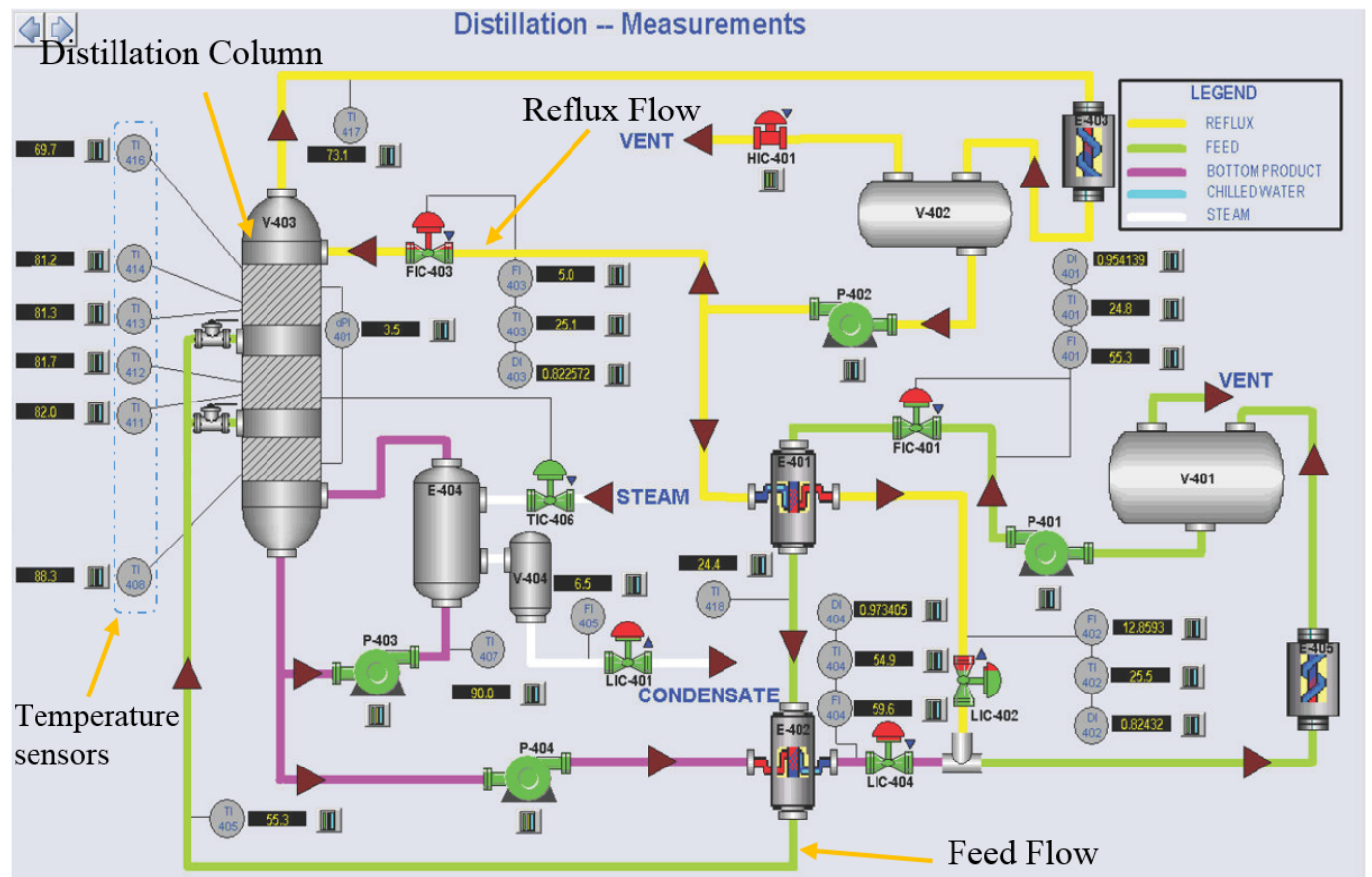

Fig. 9: Experimental setup: packed bed distillation column.
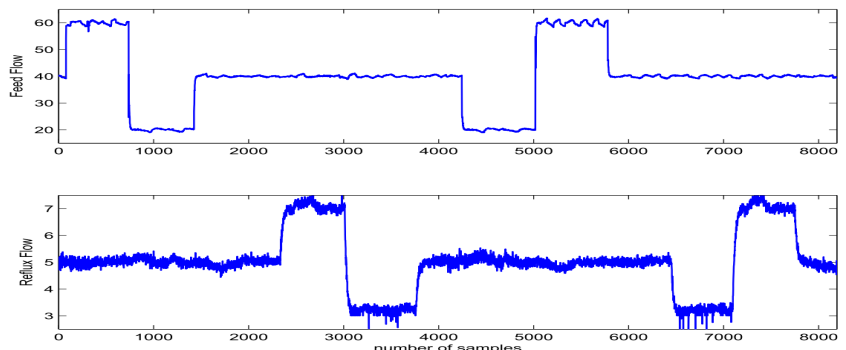

Fig. 10: Input data (feed flow and reflux flow) collected from the distillation column.

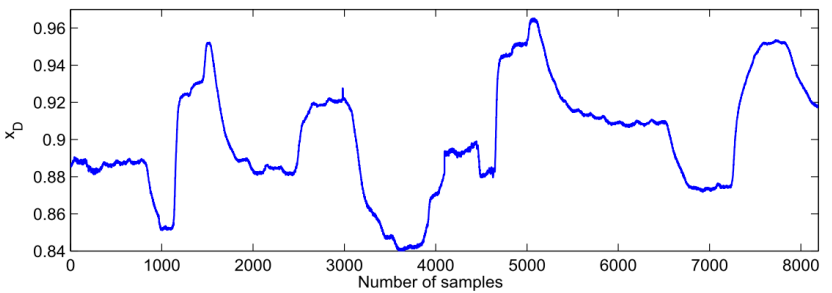

Fig. 11: Output data, xD, (i.e., methanol in distillate).

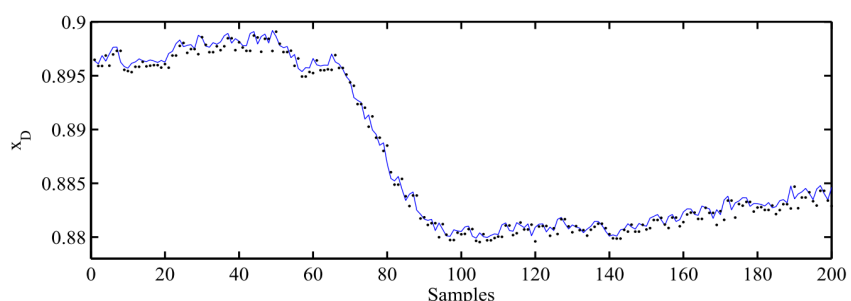

Fig. 12: Measured xD (black dots) and predicted xD (in blue) obtained from the selected PLS model.

last one of the set. Monitoring results of the fifth charts are shown in Figures 14(a)-(e). The PLS-based $T^{2}$ and $Q$ charts exhibit a very poor fault detection performance (Figures 14(a)(b)). Figures 14(c)-(e) show that the PLS-based MCUSUM, MEWMA, and CRPS-Shewhart charts detect the first signal at the 250 observation. The parameters for the MEWMA and MCUSUM monitoring charts are the same as for the first case study. The CRPS-EWMA monitoring scheme signals this fault reasonably fast at the 215 th time point. Therefore, fewer extra observations are required for the CRPS-EWMA scheme to detect this fault, compared to the other schemes. The proposed CRPS-EWMA method clearly outperform the $T^{2}, Q$, MCUSUM, MEWMA and CRPS-Shewhart methods.

As a summary, this case study demonstrates that the conventional PLS-based $T^{2}, Q$ and MCUSUM charts cannot be effective in detecting both faults, whereas the performance of the PLS-based MEWMA and CRPS-Shewhart charts perform reasonably well. Furthermore, the results show the superior performance of the CRPS-EWMA in detecting small faults.

\section{CONCLUSION}

We show that the conventional PLS-based fault detection indices do not always have a satisfactory detection performance, especially in the case of small faults. Here, we propose a simpler and more sensitive alternative to conventional PLS methods based on CRPS chart for fault detection. PLS model performed effective dimension reduction and revealed interrelationships between the input and output variables, while CRPS-based schemes (i.e., CRPS-Shewhart and CRPSEWMA) demonstrated superior detection capacity. The superior performance of this new fault detection method combining the use of PLS and CRPS metric is demonstrated via simulated distillation column data and experimental data using a packed bed distillation column. This study also shows that a better result can be obtained by applying the PLS-based CRPSEWMA scheme. 

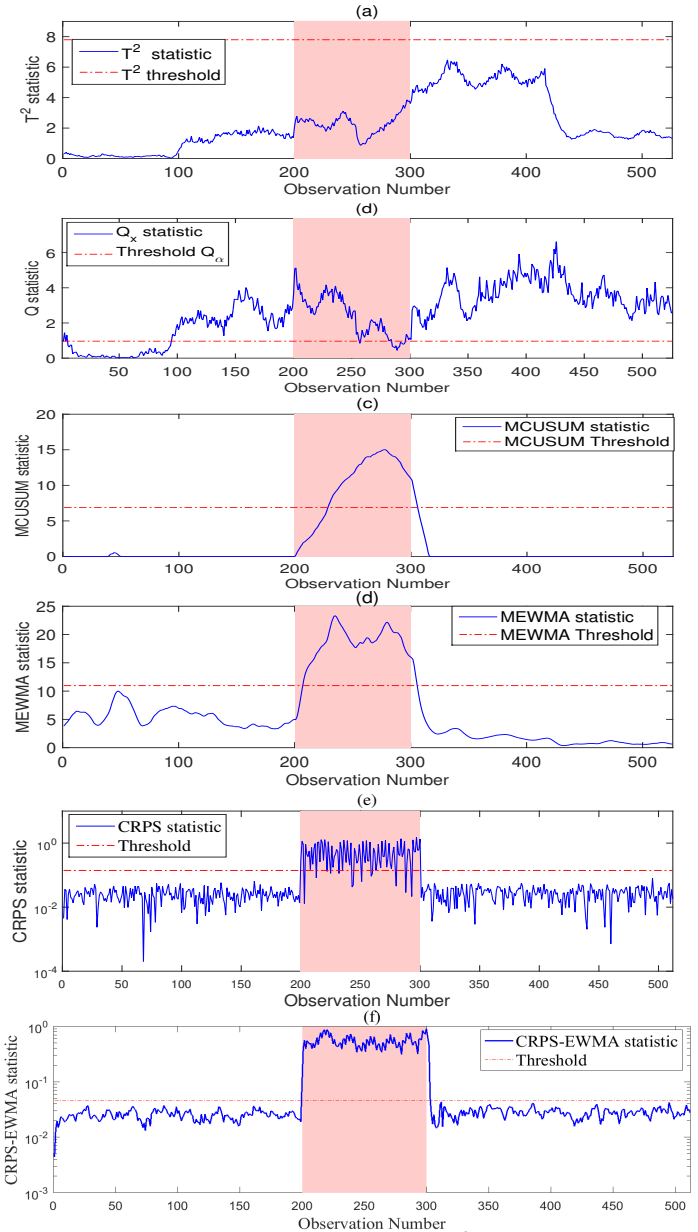

Fig. 13: Monitoring results of of the $T^{2}$ (a), $Q$ (b), MCUSUM (with $k=0.5$ and $H=6.88$ ) (c), MEWMA (with $\lambda=0.25$ ) (d), CRPS (e) and CRPS-EWMA (with $v=0.3$ ) (f) charts in the presence of bias fault in temperature measurement $T_{3}$ from samples 200 to 300 (case (a)).

Conventional multivariate fault detection methods MEWMA and such as MCUSUM rely on the assumptions of normality, independence of the data or residuals. A wavelet-based multiscale representation of data shows a good performance in separating noise from the data, approximatively decorrelating auto-correlated data and transforming the data to better follow the Gaussian distribution. It would be interesting to develop a statistical approach that can handle non-Gaussianity in the data by exploiting the benefits of the multiscale representation of data and those of the MEWMA and MCUSUM charts to better detect faults.

\section{ACKNOWLEDGMENT}

The work presented in this publication was supported by the King Abdullah University of Science and Technology (KAUST) Office of Sponsored Research (OSR) under Award No: OSR-2015-CRG4-2582.

\section{REFERENCES}

[1] J. Zhang, Y. Zhang, and Y. Guan, "Analysis of time-domain reflectometry combined with wavelet transform for fault detection in aircraft
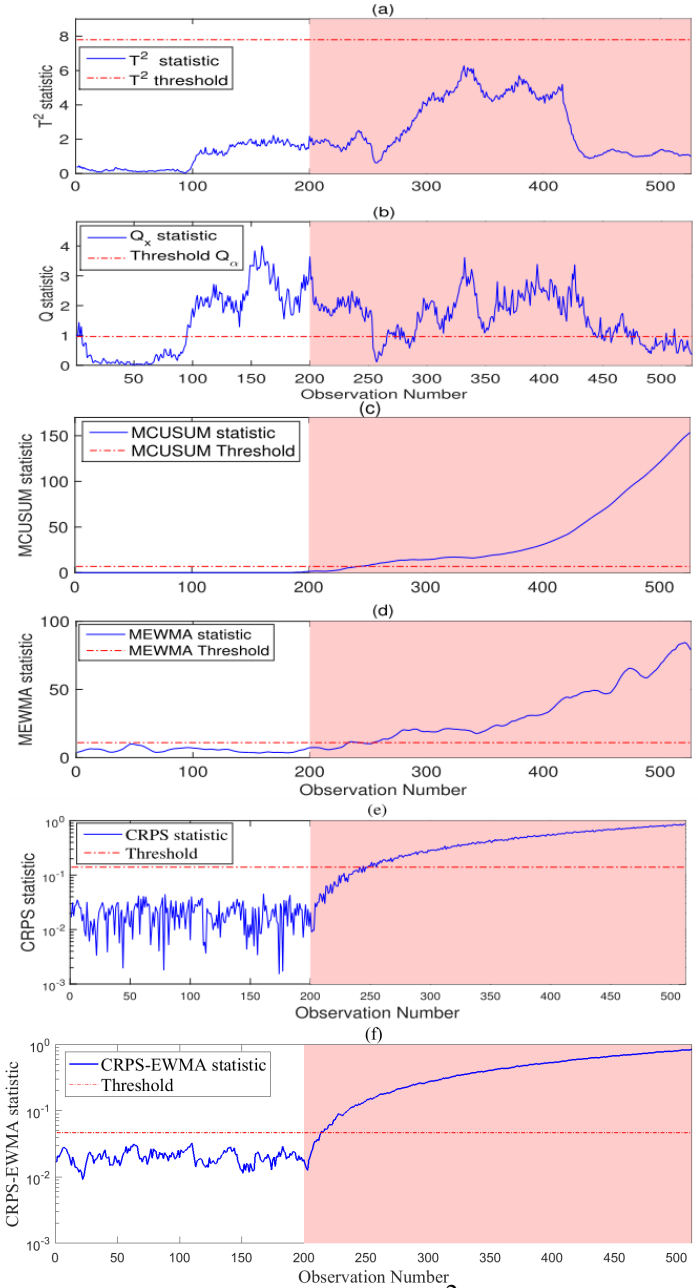

Fig. 14: Monitoring results of the $T^{2}$ (a), $Q$ (b), MCUSUM (with $k=0.5$ and $H=6.88$ ) (c), MEWMA (with $\lambda=0.25$ ) (d), CRPS-SHewhart (e) and CRPS-EWMA (with $v=0.3$ ) (f) charts in the presence of drift fault with slope 0.05 in temperature sensor $T_{3}$ from sample 200 (Case (b)).

shielded cables," IEEE Sensors Journal, vol. 16, no. 11, pp. 4579-4586, 2016.

[2] S. Yin, S. X. Ding, X. Xie, and H. Luo, "A review on basic data-driven approaches for industrial process monitoring," IEEE Transactions on Industrial Electronics, vol. 61, no. 11, pp. 6418-6428, 2014.

[3] R. Isermann, "Model-based fault-detection and diagnosis-status and applications," Annual Reviews in control, vol. 29, no. 1, pp. 71-85, 2005.

[4] F. Harrou, L. Fillatre, and I. Nikiforov, "Anomaly detection/detectability for a linear model with a bounded nuisance parameter," Annual Reviews in Control, vol. 38, no. 1, pp. 32-44, 2014.

[5] S. Zidi, T. Moulahi, and B. Alaya, "Fault detection in wireless sensor networks through SVM classifier," IEEE Sensors Journal, vol. 18, no. 1, pp. 340-347, 2018.

[6] U. Kruger and L. Xie, Advances in statistical monitoring of complex multivariate processes: with applications in industrial process control. John Wiley \& Sons, 2012.

[7] Z. Gao, S. Ding, and C. Cecati, "Real-time fault diagnosis and faulttolerant control," IEEE Transactions on Industrial Electronics, vol. 62, no. 6, pp. 3752-3756, 2015.

[8] W. Wang, "An enhanced diagnostic system for gear system monitoring," IEEE Transactions on Systems, Man, and Cybernetics, Part B (Cybernetics), vol. 38, no. 1, pp. 102-112, 2008.

[9] G. Rigatos, D. Serpanos, and N. Zervos, "Detection of attacks against power grid sensors using kalman filter and statistical decision making," IEEE Sensors Journal, vol. 17, no. 23, pp. 7641-7648, 2017. 
[10] V. Venkatasubramanian, R. Rengaswamy, K. Yin, and S. Kavuri, "A review of process fault detection and diagnosis: Part I: Quantitative model-based methods," Computers \& chemical engineering, vol. 27, no. 3, pp. 293-311, 2003.

[11] V. Venkatasubramanian, R. Rengaswamy, S. Kavuri, and K. Yin, "A review of process fault detection and diagnosis: Part III: Process history based methods," Computers \& chemical engineering, vol. 27, no. 3, pp. 327-346, 2003.

[12] S. Yin, S. Ding, a. H. H. A. Haghani, and P. Zhang, "A comparison study of basic data-driven fault diagnosis and process monitoring methods on the benchmark Tennessee Eastman process," Journal of Process Control, vol. 22, no. 9, pp. 1567-1581, 2012.

[13] S. Ding, Model-based fault diagnosis techniques: design schemes, algorithms, and tools. Springer Science \& Business Media, 2008

[14] M. Madakyaru, F. Harrou, and Y. Sun, "Improved data-based fault detection strategy and application to distillation columns," Process Safety and Environmental Protection, vol. 107, pp. 22-34, 2017.

[15] H. Yang, Y. Xia, and B. Liu, "Fault detection for T-S fuzzy discrete systems in finite-frequency domain," IEEE Transactions on Systems, Man, and Cybernetics, Part B (Cybernetics), vol. 41, no. 4, pp. 911-920, 2011.

[16] H. Paiva, R. Galvão, and T. Yoneyama, "A wavelet band-limiting filter approach for fault detection in dynamic systems," IEEE Transactions on Systems, Man, and Cybernetics-Part A: Systems and Humans, vol. 38, no. 3, pp. 680-687, 2008

[17] F. Harrou, M. Nounou, H. Nounou, and M. Madakyaru, "PLS-based EWMA fault detection strategy for process monitoring," Journal of Loss Prevention in the Process Industries, vol. 36, pp. 108-119, 2015.

[18] F. Harrou, Y. Sun, and S. Khadraoui, "Amalgamation of anomalydetection indices for enhanced process monitoring," Journal of Loss Prevention in the Process Industries, vol. 40, pp. 365-377, 2016.

[19] F. Harrou, M. Madakyaru, Y. Sun, and S. Khadraoui, "Improved detection of incipient anomalies via multivariate memory monitoring charts: Application to an air flow heating system," Applied Thermal Engineering, vol. 109, pp. 65-74, 2016.

[20] Y. Zhao, S. Wang, and F. Xiao, "Pattern recognition-based chillers fault detection method using support vector data description (SVDD)," Applied Energy, vol. 112, pp. 1041-1048, 2013.

[21] D. Agarwal and N. Kishor, "A fuzzy inference-based fault detection scheme using adaptive thresholds for health monitoring of offshore wind-farms," IEEE Sensors Journal, vol. 14, no. 11, pp. 3851-3861, 2014.

[22] Z. Wang, Q. Zhang, J. Xiong, M. Xiao, G. Sun, and J. He, "Fault diagnosis of a rolling bearing using wavelet packet denoising and random forests," IEEE Sensors Journal, vol. 17, no. 17, pp. 5581-5588, 2017.

[23] S. Yin, G. Wang, and H. Gao, "Data-driven process monitoring based on modified orthogonal projections to latent structures," IEEE Transactions on Control Systems Technology, vol. 24, no. 4, pp. 1480 - 1487, 2015.

[24] M. Madakyaru, M. Nounou, and H. Nounou, "Linear inferential modeling: theoretical perspectives, extensions, and comparative analysis," Intelligent Control and Automation, vol. 3, no. 4, pp. 376-389, 2012.

[25] G. Lee, C. Han, and E. Yoon, "Multiple-fault diagnosis of the tennessee eastman process based on system decomposition and dynamic PLS," Industrial \& engineering chemistry research, vol. 43 , no. 25 , pp. $8037-$ 8048,2004

[26] G. Wang and S. Yin, "Quality-related fault detection approach based on orthogonal signal correction and modified PLS," IEEE Transactions on Industrial Informatics, vol. 11, no. 2, pp. 398-405, 2015.

[27] W. Liang and L. Zhang, "A wave change analysis (WCA) method for pipeline leak detection using gaussian mixture model," Journal of Loss Prevention in the Process Industries, vol. 25, no. 1, pp. 60-69, 2012.

[28] H. Abdi and L. Williams, "Principal component analysis," Wiley Interdisciplinary Reviews: Computational Statistics, vol. 2, no. 4, pp. 433-459, 2010.

[29] J. Godoy, J. Vega, and J. Marchetti, "A fault detection and diagnosis technique for multivariate processes using a PLS-decomposition of the measurement space," Chemometrics and Intelligent Laboratory Systems, vol. 128, pp. 25-36, 2013.

[30] P. Geladi and B. Kowalski, "Partial least-squares regression: a tutorial," Analytica chimica acta, vol. 185, pp. 1-17, 1986.

[31] S. Qin, "Statistical process monitoring: Basics and beyond," Journal of Chemometrics, vol. 17, no. 8/9, pp. 480-502, 2003.

[32] S. Wold, H. Ruhe, H. Wold, and W. D. III, "The collinearity problem in linear regression. the partial least squares (PLS) approach to generalized inverses," SIAM Journal on Scientific and Statistical Computing, vol. 5, no. 3, pp. 735-743, 1984.
[33] J. MacGregor, C. Jaeckle, C. Kiparissides, and M. Koutoudi, "Process monitoring and diagnosis by multiblock PLS methods," AIChE Journal, vol. 40, no. 5, pp. 826-838, 1994.

[34] Q. Jia and Y. Zhang, "Quality-related fault detection approach based on dynamic kernel partial least squares," Chemical Engineering Research and Design, vol. 106, pp. 242-252, 2016.

[35] E. Grimit, T. Gneiting, V. Berrocal, and N. Johnson, "The continuous ranked probability score for circular variables and its application to mesoscale forecast ensemble verification," Ouarterly Journal of the Royal Meteorological Society, vol. 132, no. 621C, pp. 2925-2942, 2006.

[36] T. Gneiting, A.E.Raftery, A. W. III, and T. Goldman, "Calibrated probabilistic forecasting using ensemble model output statistics and minimum crps estimation," Monthly Weather Review, vol. 133, no. 5, pp. 1098-1118, 2005.

[37] F. Harrou, Y. Sun, and M. Madakyaru, "Kullback-leibler distance-based enhanced detection of incipient anomalies," Journal of Loss Prevention in the Process Industries, vol. 44, pp. 73-87, 2016.

[38] T. Kourti and J. MacGregor, "Process analysis, monitoring and diagnosis using multivariate projection methods: A tutorial," Chemometrics and Intelligent Laboratory Systems, vol. 28, no. 3, pp. 3-21, 1995.

[39] B. Li, J. Morris, and E. Martin, "Model selection for partial least squares regression," Chemometrics and Intelligent Laboratory Systems, vol. 64 no. 1, pp. 79-89, 2002.

[40] S. Yin, X. Zhu, and O. Kaynak, "Improved pls focused on keyperformance-indicator-related fault diagnosis," IEEE Transactions on Industrial Electronics, vol. 62, no. 3, pp. 1651-1658, 2015.

[41] J. Jackson and G. Mudholkar, "Control procedures for residuals associated with principal component analysis," Technometrics, vol. 21, pp. 341-349, 1979.

[42] D. C. Montgomery, "Introduction to statistical quality control," John Wiley\& Sons, New York, 2005.

[43] T. Gneiting and M. Katzfuss, "Probabilistic forecasting," Annual Review of Statistics and Its Application, vol. 1, pp. 125-151, 2014.

[44] C. Fraley, A. Raftery, and T. Gneiting, "Calibrating multimodel forecast ensembles with exchangeable and missing members using bayesian model averaging," Monthly Weather Review, vol. 138, no. 1, pp. 190202, 2010.

[45] T. Gneiting, F. Balabdaoui, and A. Raftery, "Probabilistic forecasts, calibration and sharpness," Journal of the Royal Statistical Society: Series B (Statistical Methodology), vol. 69, no. 2, pp. 243-268, 2007.

[46] T. L. Thorarinsdottir and T. Gneiting, "Probabilistic forecasts of wind speed: ensemble model output statistics by using heteroscedastic censored regression," Journal of the Royal Statistical Society: Series A (Statistics in Society), vol. 173, no. 2, pp. 371-388, 2010.

[47] S. Baran and S. Lerch, "Log-normal distribution based ensemble model output statistics models for probabilistic wind-speed forecasting," Quarterly Journal of the Royal Meteorological Society, vol. 141, no. 691, pp. 2289-2299, 2015.

[48] M. Taillardat, O. Mestre, M. Zamo, and P. Naveau, "Calibrated ensemble forecasts using quantile regression forests and ensemble model output statistics," Monthly Weather Review, vol. 144, no. 6, pp. 2375-2393, 2016.

[49] D. C. Montgomery, Introduction to statistical quality control. John Wiley \& Sons, 2007.

[50] F. Harrou and M. N. Nounou, "Monitoring linear antenna arrays using an exponentially weighted moving average-based fault detection scheme,' Systems Science \& Control Engineering: An Open Access Journal, vol. 2, no. 1, pp. 433-443, 2014.

[51] M. Madakyaru, M. Nounou, and H. Nounou, "Integrated multiscale latent variable regression and application to distillation columns," Modelling and Simulation in Engineering, vol. 2013, p. 3, 2013. 